\title{
Evaluación de la tasa de infiltración en tierras agrícolas, forestales y de pastoreo en la subcuenca del río Shullcas
}

\author{
Evaluation of the infiltration rate in farm, forestry and \\ grazing land in the Shullcas River's basin
}

Guillermo Carlos Gómez' ${ }^{1}$ Rubén Munive Cerrón'2, Tito Mallma Capcha ${ }^{3}$, Carlos Orihuela Villavicencio ${ }^{4}$ Universidad Continental

\section{RESUMEN}

Objetivos: Evaluar y determinar el uso del suelo con mayor tasa de infiltración en la subcuenca del río Shullcas en Huancayo. Métodos: Se desarrolló una investigación básica con diseño correlacional. Los usos actuales de la tierra fueron identificadas y demarcadas, según la normativa peruana de clasificación de tierras por su capacidad de uso mayor; en estas zonas se seleccionó 31 puntos de muestreo al azar usando las herramientas de software ArcGIS, en los que se midieron la infiltración empleando infiltrómetros de doble anillo, se registraron los tipos de cobertura vegetal y se tomaron muestras de suelo para analizar sus contenidos de arcilla, arena, limo y humedad; asimismo densidad aparente $y$ porosidad en laboratorio. Resultados: Las tasas de infiltración promedio, según el tipo de uso de tierras, fueron: $14,04 \mathrm{~cm} / \mathrm{h}$ para las tierras forestales; 12,42 $\mathrm{cm} / \mathrm{h}$ para las tierras agrícolas y $2,07 \mathrm{~cm} / \mathrm{h}$ para las tierras de pastoreo; además, solo el 12,63\% de la subcuenca del río Shullcas tiene infiltración mayor a $0,2 \mathrm{~cm} / \mathrm{h}$. Conclusiones: No existe diferencia estadística significativa entre la tasa de infiltración de tierras forestales y agrícolas, pero si existe diferencias de la tasa de infiltración de tierras forestales y agrícolas respecto a la tasa de infiltración de tierras de pastoreo.

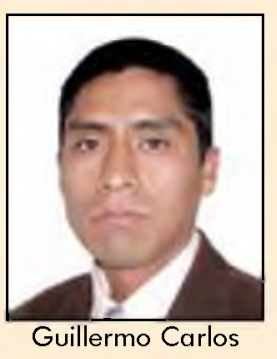

carlos.gomez.miguel@gmail.com

Historial del artículo:

Recibido: 22 de noviembre de 2013

Aprobado: 13 de febrero de 2014

Disponible en línea: 30 de junio de 2014
Palabras clave: Infiltración, cobertura vegetal, densidad aparente, porosidad, tierra agrícola, tierra forestal, tierra de pastoreo.

1 Ingeniero forestal y ambiental, investigador de la Universidad Continental.

2 Ingeniero agrónomo, M.Sc. en Suelos, docente de la Facultad de Agronomía de la Universidad Nacional del Centro del Perú.

3 Ingeniero agrícola, doctor en Recursos Hídricos, docente de la Universidad Nacional del Centro del Perú.

4 Bachiller en Ciencias Forestales y del Ambiente, especialista en Sistemas de Información Geográfica de la Empresa. 


\section{ABSTRACT}

Objectives: To evaluate and determine the land use with the highest infiltration rate into the Shullcas River's basin in Huancayo. Methods: A basic research with correlational design was developed. Existing land uses were identified and demarcated according to peruvian regulations about land classification rules for its higher use capacity; in these areas, 31 sampling points were randomly selected using the tools of ArcGIS software, in which infiltration was measured using double ring infiltrometers, the vegetation cover types were recorded and soil samples were taken to analyze the clay, sand, silt and humidity; also bulk density and porosity in laboratory. Results: The average infiltration rates, according to the land use types, were $14,04 \mathrm{~cm} / \mathrm{h}$ for forest land; $12,42 \mathrm{~cm} / \mathrm{h}$ for farmland and 2,07 cm/h for grazing land; also, only $12,63 \%$ of the Shullcas River's basin has representative infiltration greater than 0,2 $\mathrm{cm} / \mathrm{h}$. Conclusions: There is no significant statistical difference between the infiltration rate of forest and farmland, but there differences in the rate of infiltration of forest and farmland compared to the infiltration rate of grazing land.

Keywords: Infiltration, vegetation cover, bulk density, porosity, agricultural land, farmland, grazing land.

\section{INTRODUCCIÓN}

El proceso de infiltración es un componente importante del ciclo hidrológico (1), que se define como la entrada de agua hacia abajo (2), en la totalidad o parte del suelo (3), interviniendo para ello factores como: textura, presencia de costras, compactación, estructura, contenido de humedad, contenido de materia orgánica, tipo de cobertura vegetal, densidad aparente, presencia de grietas y porosidad $(3,4)$, y cuyo uso de tierras por las actividades antrópicas modifica positiva o negativamente estos factores ( 5 , $6,7)$ haciendo que la infiltración varíe y produzca impactos sobre la hidrología de una cuenca.

Numerosos estudios han demostrado que la infiltración de los suelos puede ser un buen indicador de la calidad y la salud del suelo (8), ya que al transportar agua dentro del suelo constituye como medio principal para conservar la actividad física, química y biológica de este, al mismo tiempo que recarga los acuíferos (9). Algunos resultados para el caso de tierras agrícolas revelaron que la mayor estabilidad de la estructura del suelo se relaciona con prácticas de labranza de conservación debido a que generan mayor número de macroporos y alta resistencia superficial al sellado (10).

En el ámbito internacional se han desarrollado investigaciones con el objeto de relacionar las diferentes propiedades del suelo con las prácticas de manejo realizadas, las que han servido para mejorar las técnicas de riego, minimizar la compactación del suelo, aplicar enmiendas orgánicas y realizar obras de conservación de agua como las zanjas de infiltración, surcos en contornos y otras técnicas, que conservan la humedad del suelo al mismo tiempo que aportan agua a las fuentes subterráneas $(9,6,11,5,12)$.

En el ámbito nacional y local son muy pocas las evaluaciones de las tasas de infiltración de agua a pesar de que la generación de esta información es muy valiosa para plantear y recomendar usos con fines de recarga hídrica, tecnificación de riego, incorporación de enmiendas del suelo y tipo de cubierta, posiblemente debido a que la medición directa de la infiltración es una tarea muy compleja, por ser laboriosa, lenta y costosa, dada su alta variabilidad espacial y temporal $(1,13)$, y a la técnica de medición elegida para registro de información (13).

En la subcuenca del río Shullcas se han realizado estudios por diversas instituciones sobre suelos y recursos hídricos, información que ha permitido aplicar las medidas técnicas con fines de conservación de suelos implementadas hasta la fecha $(14,15,16$, $17,18,19)$. Estos estudios han aportado 
en gran medida a conocer los suelos en términos físicos y estructurales, revelando problemas como la compactación del suelo, el establecimiento de infraestructura que impermeabiliza el suelo, las prácticas continuas de labranza y la remoción total o parcial de la cubierta vegetal del suelo. Sin embargo, debido a que los datos de tasas de infiltración en la subcuenca aún son escasos, pese a la importancia de estos, la presente investigación evalúo y determinó el uso de tierras con la tasa de infiltración más alta.

\section{MATERIAL Y MÉTODOS}

\section{Área de estudio}

La subcuenca del río Shullcas se ubica entre $11^{\circ} 52^{\prime} 41^{\prime \prime}$ a $12^{\circ} 03^{\prime} 42^{\prime \prime}$ latitud sur $y$ $75^{\circ} 02^{\prime} 16^{\prime \prime}$ a $75^{\circ} 14^{\prime} 03^{\prime \prime}$ longitud oeste, a una altitud media de $4200 \mathrm{msnm}$. Su extensión es de 218,82 km2, abarca los territorios de los distritos de El Tambo y Huancayo, pertenecientes a la provincia de Huancayo, departamento de Junín (figura 1).

\section{Delimitación de usos de tierra}

Generamos una base de datos cartográfica utilizando el software ArcGIS, a partir del cual se delimitó la subcuenca mediante el método cartográfico compuesto por la identificación de red de drenaje, delimitación de la divisoria cortando las curvas de nivel perpendicularmente ya sea por parte convexa o cóncava (20). Luego se identificó y delimitó áreas según su uso actual de tierras sobre la base del Reglamento de clasificación de tierras por su capacidad de uso mayor (21). Posteriormente se generó mapas de pendientes y de uso de tierras, identificando áreas con pendientes menores a $25 \%$ por categoría de uso; con esta información se ubicó 32 puntos de muestreo para realizar las pruebas de infiltración; obteniéndose, 7 para tierras agrícolas, 6 para tierras forestales y 19 para tierras de pastoreo.

\section{Evaluación de la infiltración}

La tasa de infiltración se midió empleando infiltrómetros metálicos de doble anillo, un cilindro externo de $50 \mathrm{~cm}$ de diámetro por

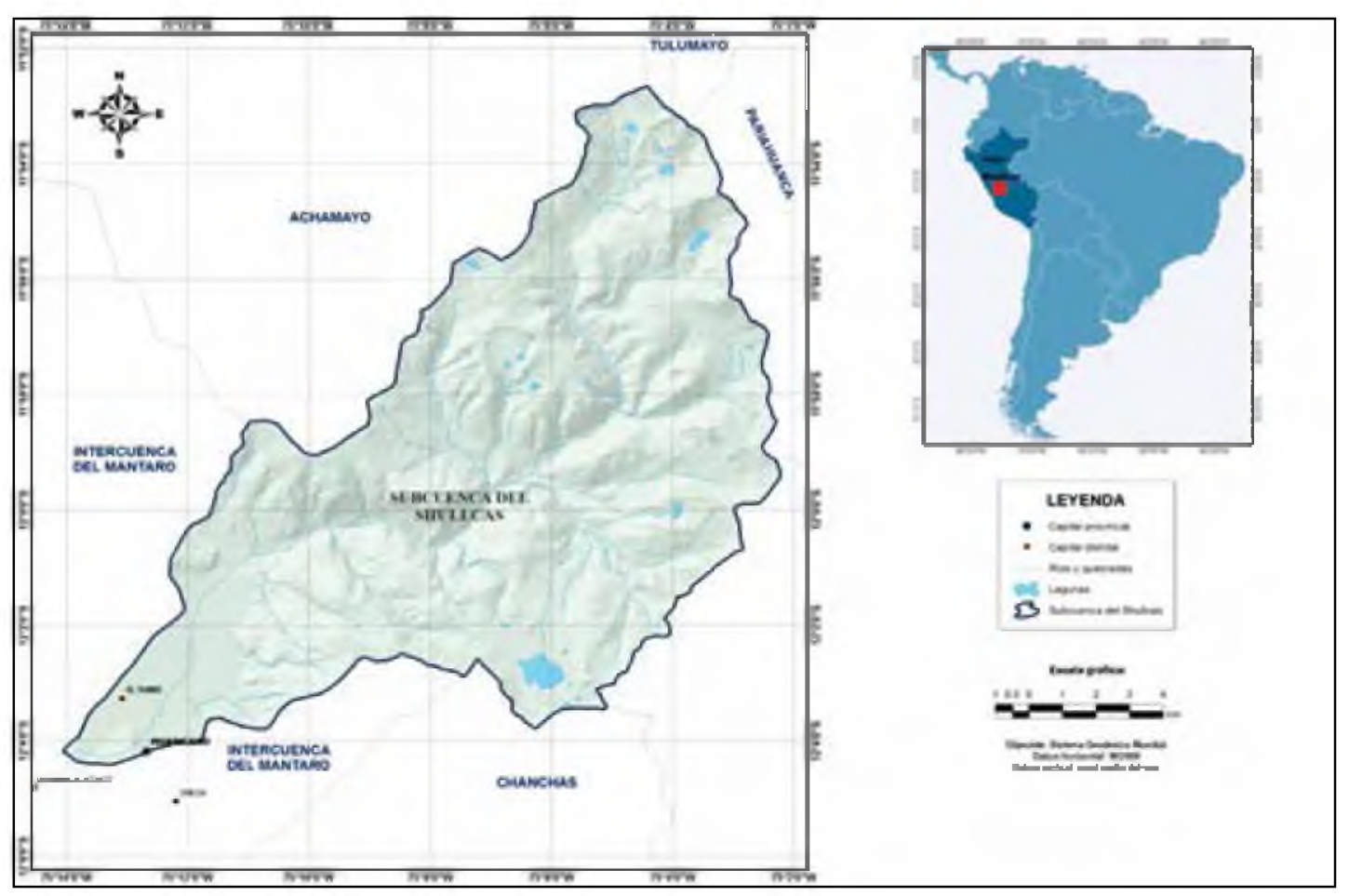

Figura $\mathrm{N}^{\circ}$ 1: Ubicación de la subcuenca del río Shullcas. 


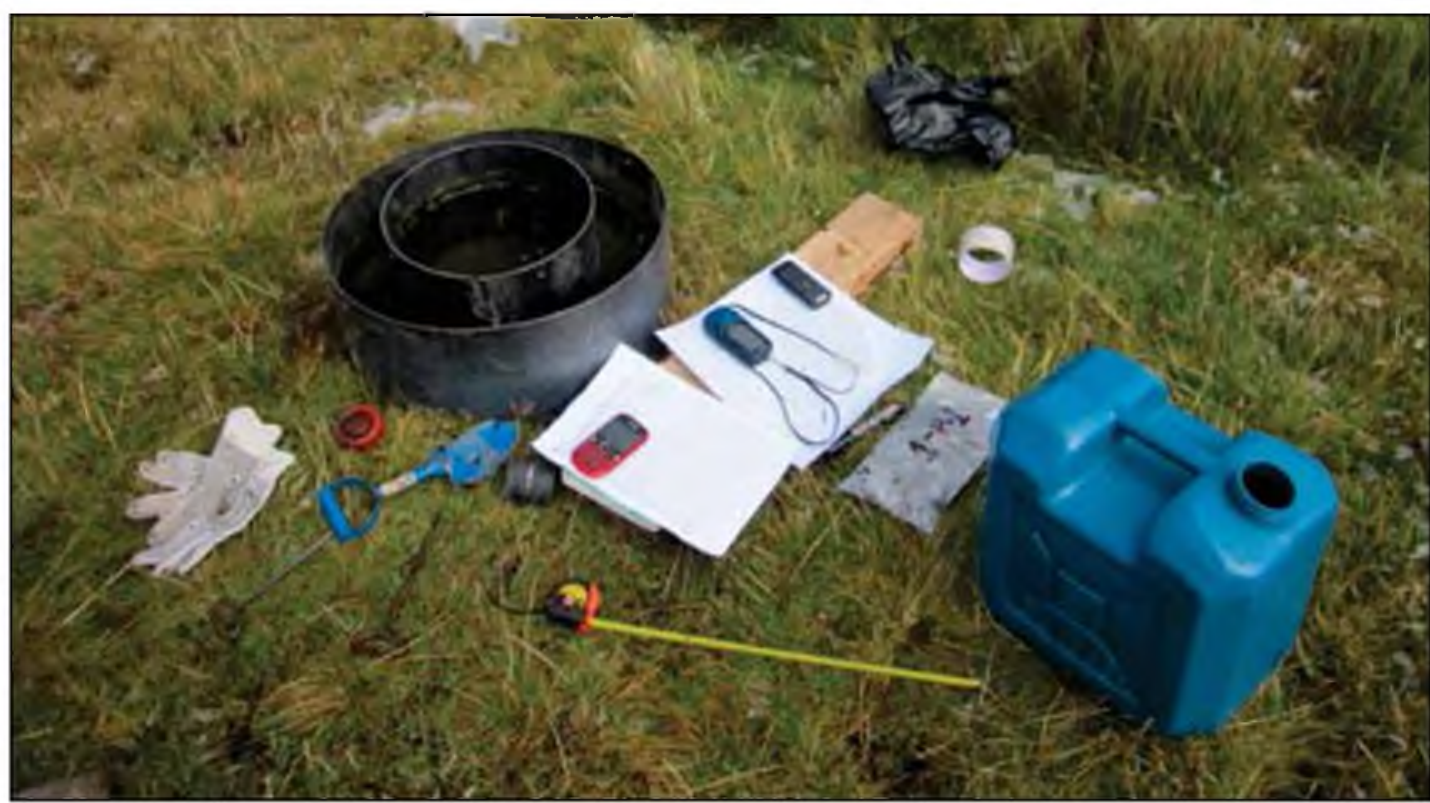

Figura $N^{\circ}$ 2: Infiltrómetro de doble anillo y materiales para la medición.

$40 \mathrm{~cm}$ de alto y un cilindro interno de 30 $\mathrm{cm}$ de diámetro por $50 \mathrm{~cm}$ de alto (figura 2). Para la instalación de los cilindros, se ubicó el punto de muestreo, se examinó la superficie del terreno para no dañar los cilindros, se evitó áreas afectadas por el tránsito de animales y maquinarias, se limpió la superficie del terreno sin retirar la cobertura vegetal, se instaló los cilindros dentro del suelo (cilindro externo $10 \mathrm{~cm}$ dentro del suelo y cilindro interno $15 \mathrm{~cm}$ dentro del suelo), se llenó agua en los cilindros y se midió la altura del nivel del agua dentro del cilindro pequeño cada 2,5 , $10,15,20,25$ y 30 minutos, considerando que el descenso del agua sea notorio entre cada medición, hasta que el descenso del nivel fue constante.

\section{Evaluación de tierras}

Se registró el tipo de cobertura vegetal presente en los puntos de muestreo

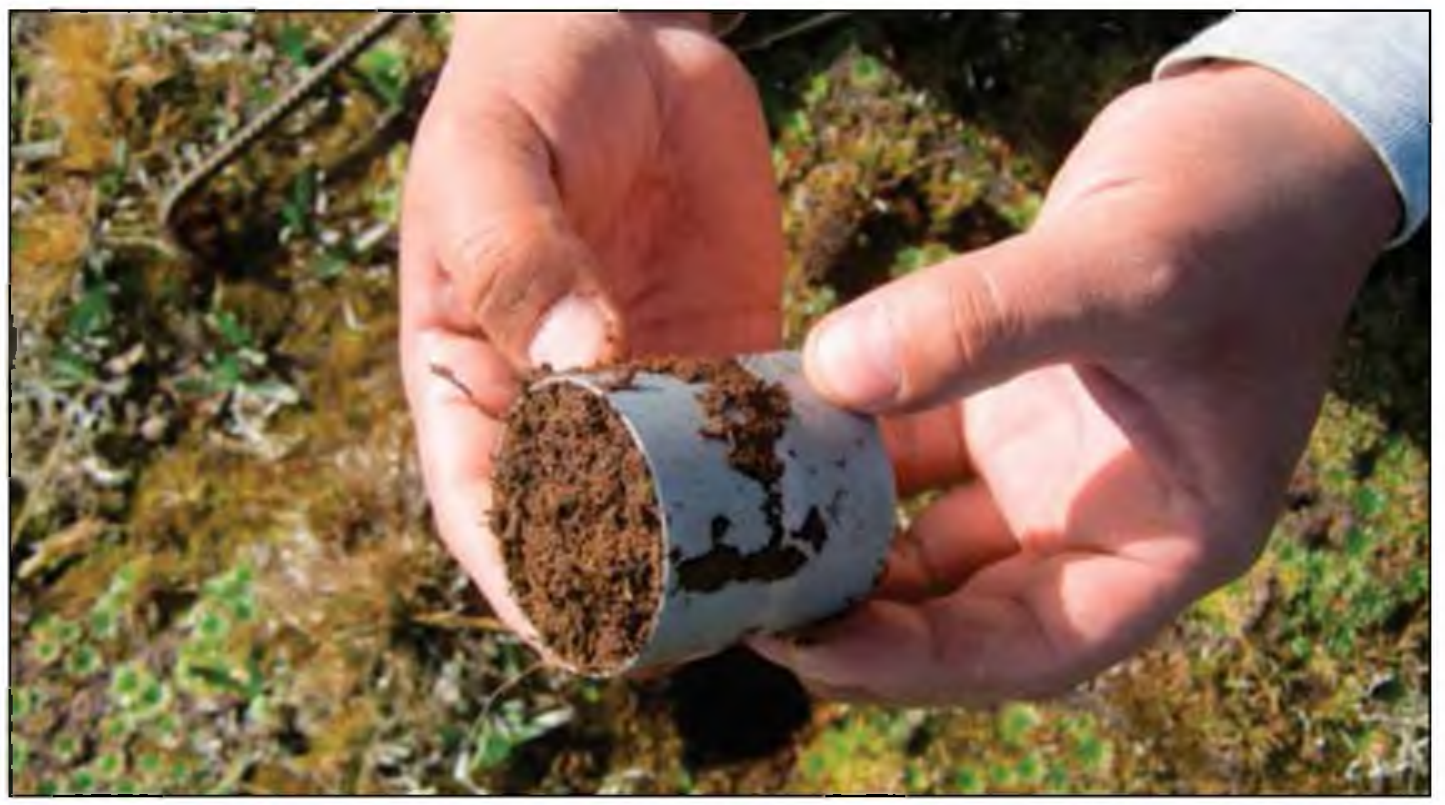

Figura $\mathrm{N}^{\circ}$ 3: Muestreo de suelo con cilindro de aluminio. 
mediante observación directa y se sacaron dos muestras de suelo por cada punto de evaluación, el primero empleando cilindros de aluminio (figura 3 ) y el segundo a través bolsas plásticas de $1 \mathrm{~kg}$, las muestras de suelo fueron llevadas al laboratorio donde se evaluó el contenido de humedad, la densidad aparente y la porosidad por el método gravimétrico; además se avaluó la textura del suelo mediante el método de Bouyoucos.

\section{Modelo de infiltración}

Se generó un modelo de infiltración basado en el contenido de arena, limo y arcilla, la densidad aparente, la porosidad, el contenido de humedad y el tipo de cobertura vegetal, que fueron correlacionados con la tasa de infiltración, obteniendo un mapa con intervalos de infiltración para la subcuenca.

Tabla $N^{\circ}$ 1: Datos registrados según tipo de uso de tierras.

\begin{tabular}{|c|c|c|c|c|c|c|c|c|c|}
\hline$N^{\circ}$ & $\begin{array}{l}\text { Tipo de } \\
\text { uso de } \\
\text { tierras } \\
\end{array}$ & $\begin{array}{c}\text { Tasa de } \\
\text { infiltración } \\
(\mathrm{cm} / \mathrm{h})\end{array}$ & $\begin{array}{c}\% \\
\text { Arena }\end{array}$ & $\begin{array}{c}\% \\
\text { Arcilla }\end{array}$ & $\begin{array}{c}\% \\
\text { Limo }\end{array}$ & $\begin{array}{c}\% \\
\text { Contenido } \\
\text { de humedad }\end{array}$ & $\begin{array}{l}\text { Densidad } \\
\text { aparente } \\
(\mathrm{g} / \mathrm{cm} 3) \\
\end{array}$ & $\begin{array}{l}\text { Porosidad } \\
\text { (\%) }\end{array}$ & $\begin{array}{c}\text { Tipo de } \\
\text { cobertura } \\
\text { vegetal } \\
\end{array}$ \\
\hline 1 & A & 1,10 & 42,88 & 26,40 & 30,72 & 16,98 & 1,29 & 48,60 & $C-E$ \\
\hline 2 & A & 29,60 & 60,88 & 12,56 & 26,56 & 7,61 & 1,33 & 50,26 & C-E3 \\
\hline 3 & A & 18,00 & 60,88 & 12,56 & 26,56 & 7,61 & 1,33 & 50,26 & C-E3 \\
\hline 4 & A & 5,50 & 42,88 & 24,40 & 32,72 & 6,44 & 1,28 & 48,45 & $\mathrm{Pc}$ \\
\hline 5 & A & 3,00 & 42,88 & 24,40 & 32,72 & 6,44 & 1,28 & 48,45 & C-E3 \\
\hline 6 & A & 8,80 & 48,16 & 13,84 & 38,00 & 10,54 & 1,32 & 49,66 & C-E3 \\
\hline 7 & A & 20,93 & 48,16 & 13,84 & 38,00 & 10,54 & 1,32 & 49,66 & $\mathrm{Pr}$ \\
\hline 8 & $\mathrm{~F}$ & 3,00 & 44,16 & 19,84 & 36,00 & 62,31 & 0,89 & 33,51 & B \\
\hline 9 & $F$ & 2,60 & 44,16 & 19,84 & 36,00 & 62,31 & 0,89 & 33,51 & B \\
\hline 10 & $\mathrm{~F}$ & 9,60 & 40,88 & 16,40 & 42,72 & 36,87 & 0,79 & 29,89 & B \\
\hline 11 & $\mathrm{~F}$ & 18,00 & 82,16 & 2,40 & 15,44 & 13,96 & 1,28 & 48,30 & B \\
\hline 12 & $\mathrm{~F}$ & 37,00 & 57,44 & 11,12 & 31,44 & 17,21 & 1,23 & 46,49 & B \\
\hline 13 & $P$ & 2,43 & 64,88 & 5,12 & 30,00 & 76,33 & 0,55 & 20,83 & $\mathrm{Pn}$ \\
\hline 14 & $P$ & 1,40 & 68,88 & 1,84 & 29,28 & 84,31 & 0,71 & 26,62 & $\mathrm{Pn}$ \\
\hline 15 & $P$ & 1,20 & 62,88 & 3,12 & 34,00 & 60,36 & 0,82 & 30,84 & $\mathrm{Pn}$ \\
\hline 16 & $P$ & 2,10 & 68,16 & 1,12 & 30,72 & 35,61 & 0,80 & 30,24 & $\mathrm{Pn}$ \\
\hline 17 & $P$ & 2,00 & 74,88 & 4,56 & 20,56 & 66,47 & 0,69 & 26,11 & $\mathrm{Pn}$ \\
\hline 18 & $P$ & 1,50 & 54,16 & 7,56 & 38,28 & 77,86 & 0,89 & 33,41 & $\mathrm{Pn}$ \\
\hline 19 & $P$ & 1,10 & 67,44 & 2,84 & 29,72 & 13,75 & 1,27 & 47,95 & $\mathrm{Pn}$ \\
\hline 20 & $P$ & 3,00 & 68,88 & 5,12 & 26,00 & 53,98 & 1,01 & 37,94 & $\mathrm{Pn}$ \\
\hline 21 & $P$ & 3,73 & 66,88 & 5,84 & 27,28 & 63,64 & 0,60 & 22,69 & $\mathrm{Pn}$ \\
\hline 22 & $P$ & 3,61 & 52,88 & 5,84 & 41,28 & 93,74 & 0,66 & 24,91 & $\mathrm{Pn}$ \\
\hline 23 & $P$ & 3,20 & 72,88 & 3,12 & 24,00 & 52,62 & 0,56 & 21,13 & $\mathrm{Pn}$ \\
\hline 24 & $P$ & 0,67 & 68,88 & 1,12 & 30,00 & 41,81 & 0,94 & 35,62 & $\mathrm{Pr}$ \\
\hline 25 & $P$ & 6,74 & 42,88 & 26,40 & 30,72 & 20,23 & 1,30 & 49,01 & $\mathrm{Pr}$ \\
\hline 26 & $P$ & 0,20 & 77,44 & 1,84 & 20,72 & 32,44 & 0,75 & 28,23 & $\mathrm{Pn}$ \\
\hline 27 & $P$ & 2,00 & 74,88 & 1,84 & 23,28 & 81,75 & 0,87 & 32,81 & $\mathrm{Pn}$ \\
\hline 28 & $P$ & 2,00 & 58,88 & 16,40 & 24,72 & 71,45 & 0,97 & 36,48 & $\mathrm{Pn}$ \\
\hline 29 & $P$ & 2,70 & 79,44 & 1,84 & 18,72 & 78,29 & 0,69 & 25,96 & $\mathrm{Pn}$ \\
\hline 30 & $P$ & 0,64 & 91,44 & 1,84 & 6,72 & 66,93 & 0,68 & 25,56 & $\mathrm{Pn}$ \\
\hline 31 & $P$ & 3,40 & 92,88 & 1,84 & 5,28 & 40,86 & 0,71 & 26,97 & $\mathrm{Pn}$ \\
\hline
\end{tabular}

Donde A significa tierra agrícola; $F$, tierra forestal; $P$, tierra de pastoreo; $C$ - $E$, cultivo recién sembrado; C-E3, cultivo en etapa de madurez; Pc, pasto cultivado; B, vegetación arbórea y/o arbustiva; $\mathrm{Pr}$, pasto residente; $\mathrm{Pn}$, pradera nativa. 


\section{RESULTADOS}

La tabla 1 resume los datos registrados en campo y obtenidos en laboratorio para los 31 puntos de muestreo.

La tabla 2 resume los valores medios de las características según el tipo de uso de tierras, donde comparativamente se aprecia que las tierras de pastoreo presentan mayor contenido de arena $(70,82 \%)$ y contenido de humedad $(59,46 \%)$, las tierras forestales mayor contenido de limo $(32,32 \%)$, y las tierras agrícolas, mayor contenido de arcilla $(18,29 \%)$, mayor densidad aparente $(1,31$ $\%)$ y mayor porosidad $(49,34 \%)$.

La tabla 3 presenta las tasas medias de infiltración según el tipo de uso de tierras, donde las tierras forestales alcanzan 14,04
La figura 4 muestra las tendencias de infiltración acumulada de acuerdo con el tiempo transcurrido, donde se aprecian todos los ensayos de infiltración realizados en campo que evidencian mayor variabilidad en tierras agrícolas y de pastoreo.

La tabla 5 muestra que las tierras agrícolas presentaron menor tiempo para alcanzar la tasa constante de infiltración (infiltración básica), seguido por las tierras forestales; y que las tierras de pastoreo demandaron mayor tiempo para alcanzar la referida tasa. Según las categorías de tasas de infiltración, las tierras agrícolas tienen una infiltración moderadamente rápida, las tierras de pastoreo una infiltración moderada y las tierras forestales una infiltración rápida (22).

Tabla N 2: Características de las tierras según tipo de uso.

\begin{tabular}{lrrr}
\hline \multicolumn{1}{c}{ Variable } & Tierra agrícola & Tierra forestal & Tierra de pastoreo \\
\hline Arena (\%) & 49,53 & 53,76 & 70,82 \\
Arcilla (\%) & 18,29 & 13,92 & 4,10 \\
Limo (\%) & 32,18 & 32,32 & 25,08 \\
Humedad del suelo (\%) & 9,45 & 38,53 & 59,46 \\
Densidad aparente (g/cm3) & 1,31 & 1,02 & 0,79 \\
Porosidad (\%) & 49,34 & 38,34 & 29,95 \\
\hline
\end{tabular}

Tabla Nº 3: : Tasa de infiltración según uso de tierras.

\begin{tabular}{|c|c|c|c|c|c|c|c|c|}
\hline \multirow[b]{2}{*}{ Uso } & \multirow[b]{2}{*}{$N$} & \multirow[b]{2}{*}{ Media } & \multirow[b]{2}{*}{$\begin{array}{l}\text { Desviación } \\
\text { típica }\end{array}$} & \multirow[b]{2}{*}{$\begin{array}{l}\text { Error } \\
\text { típico }\end{array}$} & \multicolumn{2}{|c|}{$\begin{array}{l}\text { Intervalo de confianza } \\
\text { para la media al } 95 \%\end{array}$} & \multirow[b]{2}{*}{ Mín. } & \multirow[b]{2}{*}{ Máx. } \\
\hline & & & & & $\begin{array}{l}\text { Límite } \\
\text { inferior }\end{array}$ & $\begin{array}{l}\text { Límite } \\
\text { superior }\end{array}$ & & \\
\hline Agrícola & 7 & 12,42 & 10,62 & 4,01 & 2,60 & 22,24 & 1,10 & 29,60 \\
\hline Pastoreo & 19 & 2,07 & 1,05 & 0,24 & 1,56 & 2,57 & 0,20 & 3,73 \\
\hline Forestal & 5 & 14,04 & 14,28 & 6,38 & $-3,69$ & 31,77 & 2,60 & 37,00 \\
\hline Total & 31 & 6,33 & 8,97 & 1,61 & 3,04 & 9,62 & 0,20 & 37,00 \\
\hline
\end{tabular}

$\mathrm{cm} / \mathrm{hr}$ con una desviación mayor; las tierras agrícolas, $12,42 \mathrm{~cm} / \mathrm{h}$; y las tierras de pastoreo, $2,07 \mathrm{~cm} / \mathrm{h}$.

La tabla 4 muestra que no existe diferencia estadística significativa entre la tasa de infiltración de tierras forestales y agrícolas, pero si existe diferencia estadística significativa con las tierras de pastoreo.
Tabla $N^{\circ}$ 4: Subconjuntos homogéneos de tasa de infiltración. HSD de Tukey ${ }^{a, b}$

\begin{tabular}{lrcr}
\hline & & \multicolumn{2}{c}{$\begin{array}{c}\text { Subconjunto para } \\
\text { alfa }=0,05\end{array}$} \\
\cline { 3 - 4 } $\begin{array}{c}\text { Tipo de } \\
\text { uso de } \\
\text { suelo }\end{array}$ & N & \multicolumn{1}{c}{$\mathbf{1}$} & $\mathbf{2}$ \\
\hline Pastoreo & 19 & 2,065 & \\
Agrícola & 7 & & 12,419 \\
Forestal & 5 & & 14,040 \\
Sig. & & 1,000 & 0,904 \\
\hline
\end{tabular}




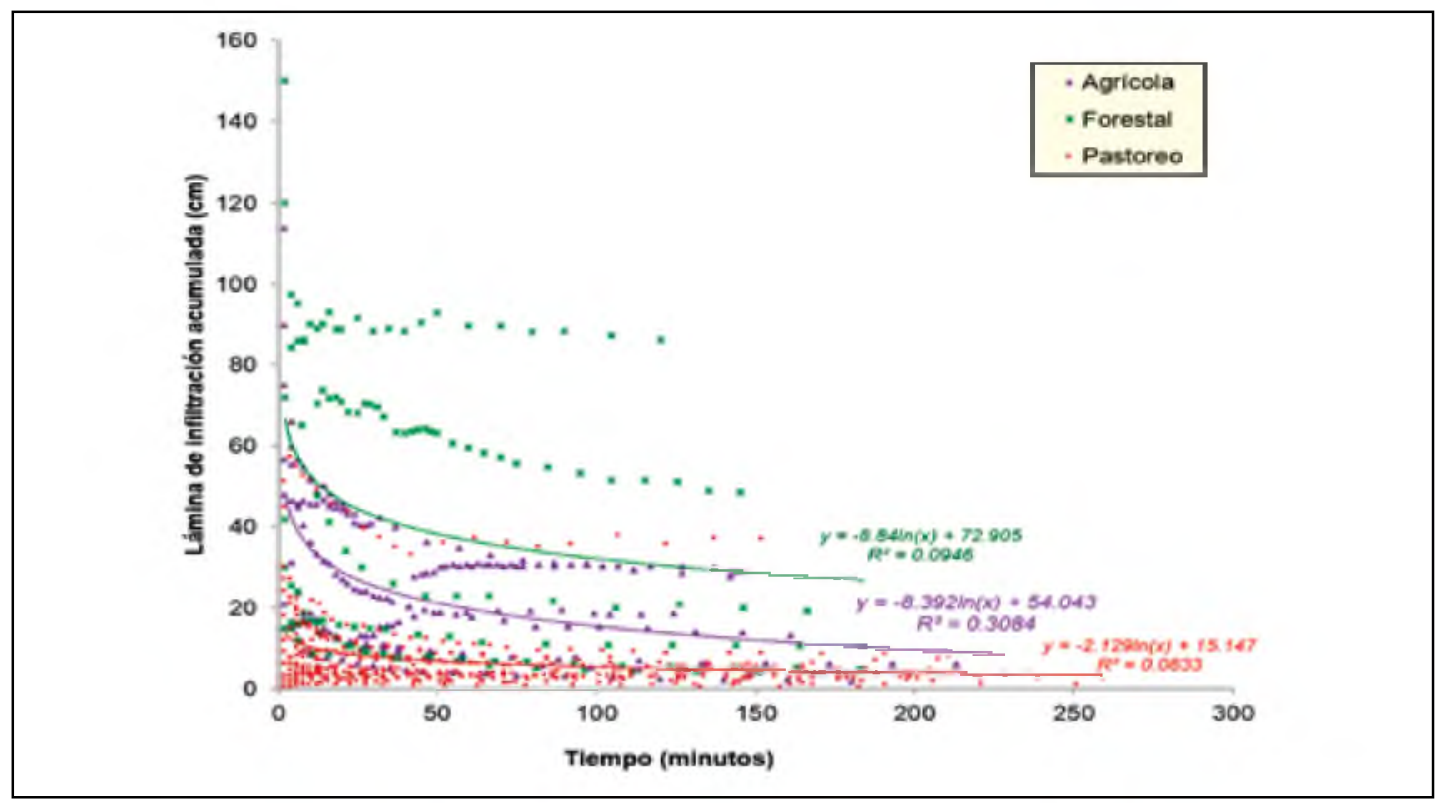

Figura $N^{\circ}$ 4: Infiltración acumulada según tipo de uso de tierra.

Las tablas 6,7 y 8 muestran el análisis del modelo de infiltración basado en regresión lineal que obtuvo un coeficiente de determinación de 0,643 , revelando que las variables predictoras que explican la tasa de infiltración (Ti) son: tipo de uso de tierras (Tu), contenido de limo (Li), contenido de arcilla (Ar), tipo de cobertura vegetal (Tc), humedad del suelo $(\mathrm{Hs})$ y porosidad (Po), excluyendo a las variables contenido de

Tabla $N^{\circ}$ 5: Tiempo medio de infiltración.

\begin{tabular}{lrrrrrr}
\hline \multicolumn{1}{c}{ Uso } & N & Media & $\begin{array}{c}\text { Desviación } \\
\text { típica }\end{array}$ & $\begin{array}{c}\text { Error } \\
\text { típico }\end{array}$ & Mín. & Máx. \\
\hline Agrícola & 7 & 169,29 & 39,538 & 14,944 & 124 & 228 \\
Pastoreo & 19 & 196,26 & 31,520 & 7,231 & 148 & 259 \\
Forestal & 6 & 161,5 & 24,793 & 10,122 & 120 & 184 \\
Total & 32 & 183,84 & 34,894 & 6,169 & 120 & 259 \\
\hline
\end{tabular}

Tabla $N^{\circ}$ 6: Resumen del modelo.

\begin{tabular}{ccccc}
\hline Modelo & $\mathbf{R}$ & R cuadrado & $\begin{array}{c}\text { R cuadrado } \\
\text { corregida }\end{array}$ & $\begin{array}{c}\text { Error típ. de } \\
\text { la estimación }\end{array}$ \\
\hline 1 & $0,708^{\circ}$ & 0,50 & 0,38 & 12,72 \\
\hline
\end{tabular}

a. Variables predictoras (constante): porosidad (\%), tipo de cobertura vegetal, limo (\%), arcilla (\%), tipo de uso de suelo, humedad del suelo (\%).

Tabla N ${ }^{\circ}$ 7: ANOVAb.

\begin{tabular}{|c|c|c|c|c|c|c|}
\hline \multicolumn{2}{|c|}{ Modelo } & $\begin{array}{l}\text { Suma de } \\
\text { cuadrados }\end{array}$ & gl & $\begin{array}{l}\text { Media } \\
\text { cuadrática }\end{array}$ & $\mathbf{F}$ & Sig. \\
\hline \multirow{3}{*}{1} & Regresión & 4055,877 & 6 & 675,980 & 4,181 & $0,005^{a}$ \\
\hline & Residual & 4042,058 & 25 & 161,682 & & \\
\hline & Total & 8097,935 & 31 & & & \\
\hline
\end{tabular}

a. Variables predictoras (constante): porosidad (\%), tipo de cobertura vegetal, limo (\%), arcilla (\%), tipo de uso de tierras, humedad del suelo (\%); b. variable dependiente: tasa de infiltración $(\mathrm{cm} / \mathrm{h})$. 
Tabla $N^{\circ}$ 8: Coeficientes ${ }^{\mathrm{a}}$

\begin{tabular}{|c|c|c|c|c|c|c|c|c|}
\hline \multirow{2}{*}{ Modelo } & \multicolumn{2}{|c|}{$\begin{array}{l}\text { Coeficientes no } \\
\text { estandarizados }\end{array}$} & \multirow{2}{*}{$\begin{array}{c}\begin{array}{c}\text { Coeficientes } \\
\text { tipificados }\end{array} \\
\text { Beta }\end{array}$} & \multirow{2}{*}{$t$} & \multirow{2}{*}{ Sig. } & \multicolumn{3}{|c|}{ Correlaciones } \\
\hline & B & $\begin{array}{l}\text { Error } \\
\text { típ. }\end{array}$ & & & & $\begin{array}{l}\text { Orden } \\
\text { cero }\end{array}$ & Parcial & $\begin{array}{l}\text { Semi- } \\
\text { parcial }\end{array}$ \\
\hline (Constante) & $-34,539$ & 22,589 & & $-1,529$ & 0,139 & & & \\
\hline Tipo de cobertura vegetal & 0,383 & 2,210 & 0,039 & 0,173 & 0,864 & 0,280 & 0,035 & 0,024 \\
\hline Tipo de uso de tierras & 9,451 & 6,097 & 0,378 & 1,550 & 0,134 & 0,231 & 0,296 & 0,219 \\
\hline Arcilla (\%) & $-0,479$ & 0,412 & $-0,233$ & $-1,162$ & 0,256 & $-0,021$ & $-0,226$ & $-0,164$ \\
\hline Limo (\%) & $-0,142$ & 0,298 & $-0,081$ & $-0,475$ & 0,639 & $-0,140$ & $-0,095$ & $-0,067$ \\
\hline Humedad del suelo (\%) & $-0,090$ & 0,151 & $-0,157$ & $-0,597$ & 0,556 & $-0,466$ & $-0,119$ & $-0,084$ \\
\hline Porosidad (\%) & 0,978 & 0,432 & 0,622 & 2,263 & 0,033 & 0,503 & 0,412 & 0,320 \\
\hline
\end{tabular}

a. Variable dependiente: tasa de infiltración $(\mathrm{cm} / \mathrm{h})$.

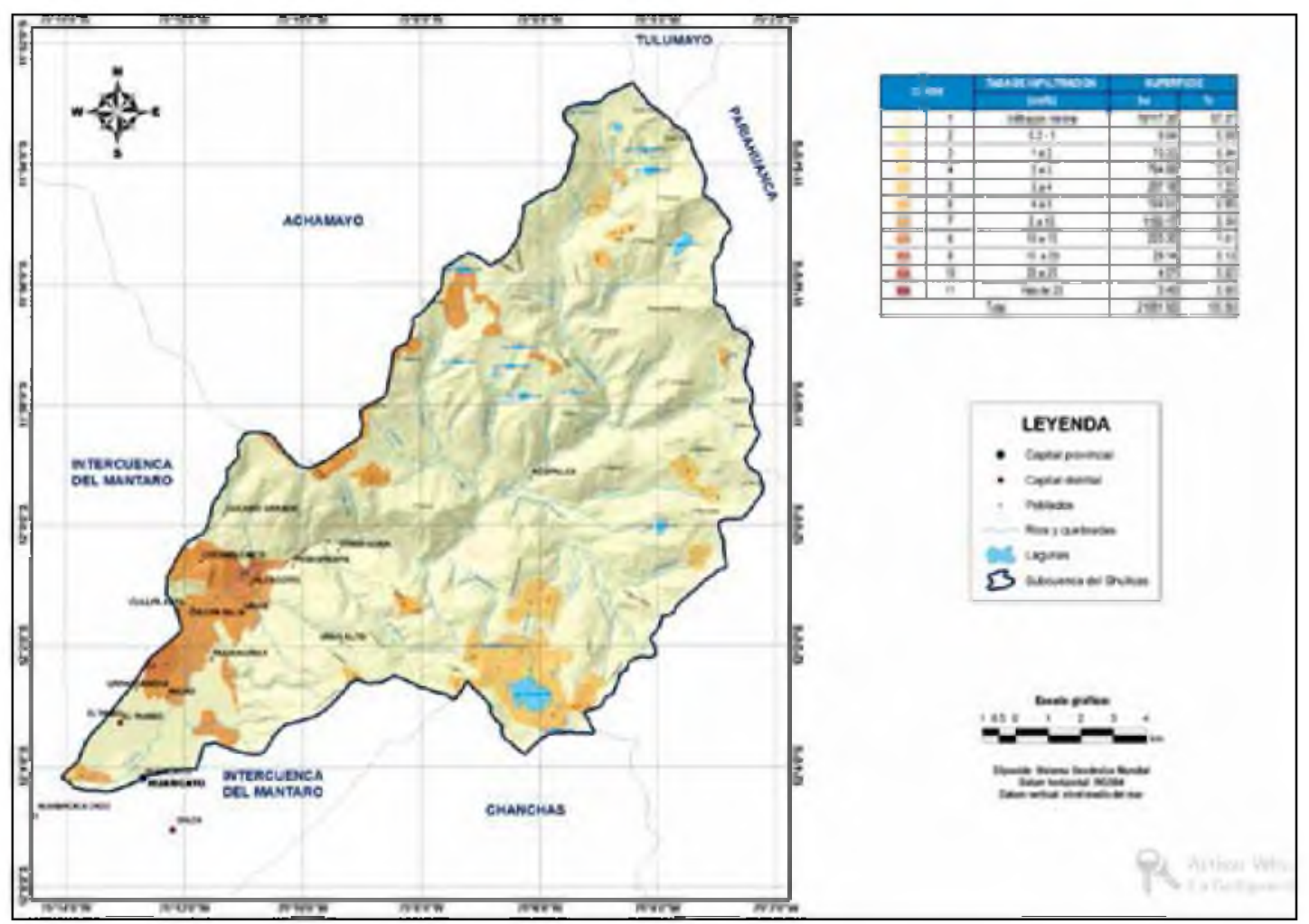

Figura $\mathrm{N}^{\circ}$ 5: Mapa de infiltración de la subcuenca del río Shullcas.

arena y densidad aparente. Su expresión matemática es: $\mathrm{Ti}=-14,194-0,289 \mathrm{Tc}+$ 4,487 Tu - 0,187 Ar + 0,076 Li - 0,094 Hs $+0,463$ Po $+0,978$ Po a partir de la cual el mapa de infiltración de la subcuenca se muestra en la figura 5.

\section{DISCUSIÓN}

Las tierras forestales presentaron una tasa de infiltración básica promedio de 14,04cm/h alcanzada en un tiempo promedio de 169,8 minutos, las tierras agrícolas presentaron una tasa de infiltración básica promedio de 12,42 cm/h alcanzada en un tiempo promedio de 169,29 minutos y las tierras de pastoreo presentaron una tasa de infiltración básica promedio de 2,07 $\mathrm{cm} / \mathrm{h}$ alcanzada en un tiempo promedio de 196,26 minutos. Los principales factores que influyeron en la infiltración fueron; tipo de uso de tierras, tipo de cobertura 
vegetal, contenido de arcilla, contenido de limo, humedad del suelo y porosidad. Así también el 12,63\% de la subcuenca del río Shullcas presentó una infiltración mayor a $0,2 \mathrm{~cm} / \mathrm{h}$.

Las tierras forestales presentaron una infiltración rápida (22), debido a que las plantaciones instaladas (19) han mejorado la estructura del suelo facilitando el movimiento del agua dentro del suelo (23, $24,25)$. Así también, la presencia de capas de hojarasca sobre la superficie del suelo del bosque retarda el escurrimiento de la superficie y proporciona más tiempo para que el agua se infiltre en el suelo.

Las tierras agrícolas presentaron una infiltración moderadamente rápida debido a que su estructura es constantemente alterada, presentando rotaciones de su cobertura vegetal con diversos cultivos como maíz, olluco, oca, trigo, cebada, arveja, papa, habas, hortalizas y alfalfa, y que en determinadas épocas del año se encuentra en descanso (19), ello influye en la cantidad de grietas causadas por los diversos cultivos, movimiento de tierra y la desecación de la arcilla (26). La infiltración moderadamente rápida de las tierras agrícolas permite que éstos demanden mayor cantidad de agua y demanden riego localizado o riego por aspersión para mantener la producción de los cultivos; además la instalación de cultivos semiperennes, incorporación de materia orgánica mediante guano, abonos verdes y compost, o el abandono temporal de la actividad $(27,28)$, ello con fines de la recuperación del suelo y obtención de tasas óptimas de infiltración entre $2 \mathrm{~cm} / \mathrm{h}$ y $6 \mathrm{~cm} / \mathrm{h}(22)$.

Las tierras de pastoreo presentaron la menor tasa de infiltración básica promedio y alcanzaron un tiempo mayor en comparación con las tierras agrícolas y forestales, por lo cual se encuentra dentro de la categoría de infiltración moderada (22). Esto se debe a que presentan una cobertura vegetal constituida mayormente por gramíneas del tipo pajonal que forman matas gruesas y altas $(<1 \mathrm{~m})$, con hojas punzocortantes cuando están maduras; asociadas a gramíneas, pegadas al piso tipo césped, usados para el pastoreo de alpacas, llamas, ovinos y vacunos, en forma continua y simultánea, sin ningún criterio técnico; donde se práctica comúnmente quema del pajonal con la finalidad de aprovechar los rebrotes (19); dichas prácticas reduce la tasa de infiltración en estas tierras (29 30), ya que se

debilita la estructura y se obstruyen los poros por la carga animal y la quema (9). Broersma et al (30) menciona que el pastoreo tiene un efecto pronunciado sobre la densidad aparente del suelo, la porosidad, la infiltración, almacenamiento de agua, las características de transporte de agua y la escorrentía; y Hillel (31) afirma que la compactación reduce los poros del suelo que resulta en una disminución de la tasa de infiltración.

Las variaciones de la cantidad de arcilla y limo de las diferentes muestras de suelo según el tipo de uso de tierras, influyó en la regulación de la infiltración debido a que son partículas que tienen menor tamaño pero mayor espacio poroso (32). Srinivasan y Poongothai sostienen que las tasas de infiltración inicial y constante son más altas debido al hecho de que tiene textura gruesa y grandes espacios porosos que promueven la infiltración rápida (33). Así también el tipo de cobertura vegetal, influyó directamente en el movimiento del agua dentro del suelo debido a su naturaleza, el tipo de vegetación que la conforma y el espesor de la misma, donde su remoción reduce la tasa de infiltración hasta en un $80 \%$ (34), debido a que su destrucción favorece el sellado superficial e inhibe la rápida filtración de agua (35). El tipo de uso de tierras, así como el cambio de uso (36), modifica completamente las características del suelo, variando la estructura interna y obstruyendo o incrementando la presencia de poros y macroporos, ya que la infiltración es rápida a través de grandes poros continuos en la superficie del suelo y frena cuando los poros se hacen más pequeños (33), los cambios en cobertura vegetal producto del tipo de uso de tierras modifica la permeabilidad del suelo, al mismo tiempo que el almacenamiento de 
agua después de la precipitación (37). El contenido de humedad del suelo regula el tiempo para alcanzar la tasa de infiltración básica, es decir, a mayor contenido de humedad, se necesita mayor tiempo para alcanzar la infiltración básica; del mismo modo, tal como señala Srinivasan y Poongothai, cuanto menor sea el contenido inicial de humedad del suelo, la tasa de infiltración instantánea será más alta (33).

Respecto al 12,63\% del área de la subcuenca que presenta infiltración representativa (mayor de $0,2 \mathrm{~cm} / \mathrm{h}$ ), se debe a que el $87,37 \%$ se encuentra en zonas con presencia de rocas, suelos muy superficiales y con pendientes pronunciadas mayores a $30 \%$, donde no se realizaron pruebas de infiltración dado a que, según la revisión bibliográfica, estas características imposibilitan en gran medida que se lleve a cabo el fenómeno de "infiltración" y en vez de ello tienen alta escorrentía superficial $(38,39)$. Janeau et al $(40)$ refiere que no hay ninguna información procedente de estudios sobre cómo varían las tasas de infiltración con el grado de pendiente, y en especial para pendientes superiores a $4 \%$ (41). Algunos estudios mencionan que existe una relación positiva entre la pendiente y la tasa de infiltración debido a que las pendientes pronunciadas previenen la formación y desarrollo de sellos superficiales (42), llegando a encontrar tasas de infiltración entre $0,1 \mathrm{~cm} / \mathrm{h}$ y 10,7 $\mathrm{cm} / \mathrm{h}$ para un suelo arcilloso y pedregoso (40). Estas zonas no fueron motivo de investigación debido a que no presentan uso alguno y son catalogados, según el reglamento de clasificación de tierras, como tierras de protección.

\section{Agradecimientos}

A la Universidad Continental por la subvención para la realización del estudio. También a Arturo Anco Arroyo, Miguel Varillas Huaynalaya, José Castañeda Cahuana, Tony Yauri Inga, Juan Espinoza Ruiz, Yerrsu Araujo Cárdenas, Alex Mucha Mucha, Deyvis Hinostroza Zárate y Jhonathan Ávila Gutarra por brindar su apoyo en la recopilación de datos.

\section{REFERENCIAS BIBLIOGRÁFICAS}

1. Parchami-Araghi F, Majid S, Ghorbani $S$, Hossein M. Point estimation of soil water infiltration process using Artificial Neural Networks for some calcareous soils. Journal of Hydrology. 2013; 481: 35-47.

2. Richards LA. Report of the Subcommittee on permeability and infiltration, Committee on Terminology. Soil Science Society of America. 1952; 16: 85-88.

3. Hillel D. Environmental Soil Physics. San Diego: Academic Press; 1998.

4. USDA [Internet]. Washington: Natural Resouces Conservation Service. 1998 [Citado el 10 de junio de 2013]. Soil quality indicators: Infiltration [2 páginas]. Disponible en: http:// soils.usda.gov/sqi/publications/files/ Infiltration.pdf

5. Akintoye $\mathrm{O}$, Ukata $\mathrm{S}$, Esomonye, $\mathrm{H}$. The effects of landuse on the infiltration capacity of coastal plain soils of Calabar-Nigeria. International Journal of Applied Science and Technology. 2012; 2(2): 80-84.

6. Návar J, Synnott T. Soil infiltration andland use in Linares. Terra Latinoamericana. 2000; 18 (003): 255262.

7. Wood MK, Blackburn W. Grazing systems: their influence on infiltration rates in the rolling plains of Texas. Journal of Range Management.1981; 34(4): $331-335$.

8. Klingebiel AA, Montgomery PH. Landcapability classification. Washington: Soil Conservation Service United States Department of Agriculture; 1961.

9. Rawls W, Ahuja L, Brakensiek D, Shirmohammadi A. Infiltration and soil water movement. Texas: Maidment DR; 1993.

10. Wuest SB. Earthworm, infiltration, and tillage relationships in a dry land peawheat rotation. Applied Soil Ecology. 2001; 18(2): 187-192.

11. Makungo R, Odiyo J. Determination of steady state infiltration rates for different soil types in select areas of Thulamela Municipality, South Africa. 
15th South African National Hydrology Symposium. Grahamstown: Rhodes University; 2011. Disponible en: http://www.ru.ac.za/static/institutes/ iwr/SANCIAHS/2011/the.pdffiles/R Makungo_Paper.pdf

12. Harden $\bar{C}$, Delmas P. Infiltration on mountain slopes: a comparison of three environments. Geomorphology. 2003; (55): 5-24.

13. Allison LE, Bernstein L, Bower CA, Brown JW, Fireman $M$, Hatcher JT, et al. Diagnosis and improvement of saline and alkali soils. Washington: United States Department of Agriculture; 1954.

14. Servicio Nacional de Meteorología e Hidrología del Perú. Los cambios del clima y sus impactos en la disponibilidad hídrica y principales cultivos en la subcuenca del río Shullcas, Junín. Lima: SENAMHI/Zona Comunicaciones; 2012.

15. Guerrero J. Estudio agrometeorológico de la subcuenca del río Shullcas. Huancayo: Proyecto de adaptación al impacto del retroceso acelerado de glaciales en los Andes Tropicales/ SENAMHI; 2012. Contrato $\mathrm{N}^{\circ}$ : 046-2012-SGCAN.

16. Servicio Nacional de Meteorología e Hidrología del Perú. Disponibilidad hídrica actual y futura al 2030 subcuenca Shullcas - Junín. Lima: Proyecto de Adaptación al Impacto del Retroceso Acelerado de Glaciales en los Andes Tropicales - PRAA/SENAMHI; 2011.

17. Administración Local del Agua Mantaro. Evaluación de recursos hídricos superficiales en la cuenca del río Mantaro. Lima: Autoridad Nacional del Agua/Ministerio de Agricultura y Riego; 2010.

18. Martínez A. Análisis de la vulnerabilidad ante los efectos del cambio climático y propuestas de adaptación en la ciudad de Huancayo. Lima: Fondo Editorial del Seminario Permanente de Investigación Agraria (SEPIA); 2007.

19. Dirección general de estudios y proyectos de recursos naturales. Caracterización de los recursos naturales renovables para el alivio a la pobreza en sierra - microcuenca Shullcas, Junín. Lima: Instituto Nacional de Recursos Naturales; 1997.

20. Intendencia de Recursos Hídricos. Delimitación y codificación de unidades hidrográficas del Perú. Lima: Ministerio de Agricultura; 2007.

21. Ministerio de Agricultura. Reglamento de clasificación de tierras por su capacidad de uso mayor. Lima: Dirección General de Asuntos Ambientales Agrarios; 2009. Decreto Supremo N 017-2009-AG

22. Food and Agriculture Organization. Colección FAO: Capacitación: Métodos sencillos para la acuicultura [CD-ROM]. Roma: FAO; 2003.

23. Landon JR. Tropical soil manual booker agriculture. Londres: International Limited; 1984.

24. Wahren A, Feger $K H$, Schwärzel K, Münch A. Land-use effects on flood generation-considering soil hydraulic conductivity measurements in modeling. Adv. Geosci. 2009; 21 : 99-107.

25. Mapa RB. Effect of reforestation using Tectona grandis on infiltration and soil water retention. Forest Ecology and Management. 1995; 77(1-3): 119. 125.

26. Mann L, Tolbert V. Soil sustainability in renewable biomass plantings. $\mathrm{AMBIO}$ : A Journal of the Human Environment. 2000; 29(8): 492-498.

27. Selim T. The effect of land use on soil infiltration rate in a heavy clay soil in Egypt. VATTEN. 2011; 67: 161-166.

28. Olson N, Gulliver J, Nieber J, Kayhanian M. Remediation to improve infiltration into compact soils. Journal of Environmental Management. 2013; 117:85-95.

29. Egbai O, Uquetan I, Ewa E, Ndik E. Infiltration rate assessment of coastal plain (ultisols) soils for sustainable crop production in the frontiers of Calabar-Nigeria. Journal of Sustainable Development. 2011 ; 4(4): 222-229.

30. Martin D, Moody J. Comparison of soil infiltration rates in burned and unburned mountainous watersheds. Hydrological Processes. 2001; 15(15): 2893-2903.

31. Broersma K, Robertson J, Chanasyk D. Effect of different cropping systems on 
soil water properties of a Boralf soil. Communication in Soil Science and Plant Analysis. 1995; 26(11-12): 17951811.

32. Hillel D. Introduction to soil physics. New York: Academic Press Inc; 1982.

33. Srinivasan K, Poongothai S. Assessment of Infiltration rate of a tank irrigation watershed of Wellington reservoir Tamilnadu. American Journal of Engineering Research. 2013; 2(7): 41 48.

34. Suárez E, Arcos E, Moreno C, Encalada $A C$, Álvarez M. Influence of vegetation types and ground cover on soil water infiltration capacity in a high-altitude páramo ecosystem. Avances en ciencias e ingenierías. 2013; 5(1): 14-21.

35. Bassey E, Imoke D, Comfort $O$. Evaluation of the infiltration capacity of soils in Akpabuyo Local Government area of cross river. Journal of Geography and Geology. 2011 ; 3(1): 189-199.

36. Neris J, Jiménez C, Fuentes J, Morillas $G$, Tejedor M. Vegetation and landuse effects on soil properties and water infiltration of andisols in Tenerife (Canary Islands, Spain). Catena. 2012; 98: 55-62.

37. Huang J, Wu $P$, Zhao $X$. Effects of rainfall intensity, underlying surface and slope gradient on soil infiltration under simulated rainfall experiments. Catena. 2013; 104: 93-102.

38. Abu-Hashim MSD. Impact of landuse and land-management on the wáter infiltration capacity of soils on a catchment scale. [Tesis doctoral]. Braunschweig: Technischen Universität Carolo - Wilhelmina; 2011.

39. Harden C, Delmas P. Infiltration on mountain slopes: a comparison of three environments. Geomorphology. 2003; (55): 5-24.

40. Janeau J, Bricquet J, Planchon $O$, Valentin C. Soil crusting and infiltration on steep slopes in northern Thailand. European Journal of Soil Science. 2003; 54: 543-553.

41. Wilcox B, Wood M, Tromble J. Factors influencing infiltrability of semiarid mountain slopes. Journal of Range Management. 1988; 41(3): 197-206.
42. Bamutaze $Y$, Makooma $M$, Gilbert $M$, Vanacker V, Bagoora $F$, Magunda $M$, et al. Infiltration characteristics of volcanic sloping soils on Mountain Elgon, Eastern Ugada. Catena. 2010; 80(2): 122-130. 